\title{
A SIMPLIFICATION IN THE CONDITIONS FOR THE ZERO-LENGTH-SPRING SEISMOGRAPH*
}

\author{
By Lucten J. B. LaCoste
}

Abstract. It has been found that for the zero-length-spring seismograph recently described the length of the arm need not equal the distance from the pivot to the point of attachment of the spring. The proof of the infinite period is given for the more general case, and the effect on the period of slight deviations from the conditions for infinite period is shown.

\section{INTRODUCTION}

Recently a single-spring vertical motion seismograph of theoretically infinite period for all positions of the seismograph arm was described. ${ }^{1}$ The present paper gives a simplification in the conditions to be met by this type of seismograph.

\section{Proof of the Infinite Period}

The seismograph described in the previous article is shown in Figure 1 if the two distances $a$ and $b$ are equal. The infinite period exists, however, even if $a$ and $b$ are not equal; this can be shown as follows:

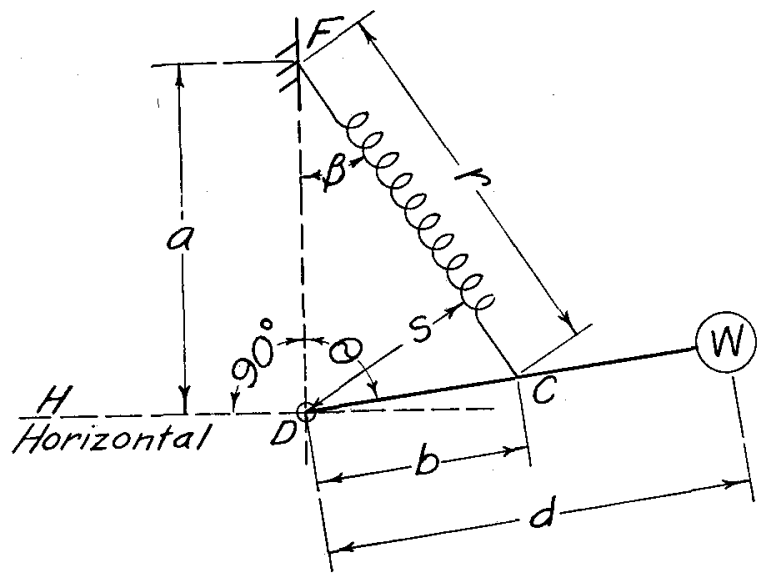

FIG. 1.-Diagram of seismograph

* [Received for publication August 5, 1934.]

1 L. J. B. LaCoste, Physics, 5, 178, 1934. 
The weight $W$ is fixed to the $\operatorname{arm} D C$. which is pivoted at $D$. The spring $F C$ is attached to the frame at $F$ and to the arm at $C$. The point of attachment $F$ of the spring is vertically above the pivot $D$. As described in the previous article the spring has an initial length (actual physical length minus elongation) of zero. In other words its actual physical length is equal to its elongation. The method of winding such a spring is given in the preceding article.

The torque produced by the weight is

$$
T_{w}=W d \sin \vartheta
$$

Since the spring has a zero initial length, the torque produced by the spring is

$$
T_{s}=-k r s
$$

when $k$ equals the spring constant.

By the sine law

and since $s$ can be expressed as

$$
r=\frac{b \sin \vartheta}{\sin \beta}
$$

$$
s=a \sin \beta
$$

the expression for $T_{s}$ becomes

The total torque is

$$
T_{s}=-k a b \sin \vartheta
$$

If

$$
\begin{aligned}
T_{0} & =T_{w}+T_{s} \\
& =(W d-k a b) \sin \vartheta
\end{aligned}
$$

then

$$
W d=k a b
$$

$$
T_{\mathrm{o}}=0
$$

for all values of $\vartheta$, and the period is infinite for all positions of the arm.

The Effect of Slight Deviations from the Conditions FOR INFINITE PERIOD

The effect on the period of varying the angle $F D H$ slightly or of adding a small initial length to the spring can be found by the same method that was used in the preceding paper. Assume that the angle $F D H$ is $90^{\circ}+\Delta$ and that the initial length of the spring is $L$; then the total torque becomes

$$
T_{\mathrm{o}}=W d \sin (\vartheta+\Delta)-k a b \sin \vartheta+L k a \sin \beta
$$


By the sine law

$$
\sin \beta=\frac{b \sin \vartheta}{r}
$$

Substituting this value of $\sin \beta$ in Equation 1 gives

when

$$
T_{\mathrm{o}}=W d \sin (\vartheta+\Delta)-k a b\left(1-\frac{L}{r}\right) \sin \vartheta
$$

For equilibrium

$$
r=\sqrt{a^{2}+b^{2}-2 a b \cos \vartheta}
$$

$$
T_{\mathrm{o}}=0
$$

and

$$
W=\frac{k a b(1-L / r) \sin \vartheta}{d \sin (\vartheta+\Delta)}
$$

The square of the frequency of vibration is

$$
F^{2}=-K^{2} \frac{d T_{0}}{d \vartheta}
$$

when $K$ is a constant.

By differentiating Equation 2 and noting that $r$ is given by Equation 3, it is found that

$$
\begin{aligned}
F^{2}= & K^{2}\{-W d \cos (\vartheta+\Delta)+k a b(1-L / r) \cos \vartheta+ \\
& \left.\frac{L k a^{2} b^{2} \sin ^{2} \vartheta}{r^{3}}\right\}
\end{aligned}
$$

Substituting the value of $W$ given in Equation 4 gives

$$
\begin{aligned}
F^{2}= & K^{2} k a b\left\{(1-L / r) \cos \vartheta-\frac{(1-L / r) \sin \vartheta}{\tan (\vartheta+\Delta)}+\right. \\
& \left.\frac{L a b \sin ^{2} \vartheta}{r^{3}}\right\}
\end{aligned}
$$

Let $^{2}$

$$
\vartheta=90^{\circ}-\delta
$$

Then, if the $\operatorname{arm} D C$ is approximately horizontal, $\delta$ is a small angle.

2 It should be noted that the $\vartheta$ in this paper is $180^{\circ}$ minus the $\vartheta$ of the preceding paper. 
Neglecting third-order powers of $\delta$ and $\Delta$

$$
\begin{aligned}
\sin \vartheta & =1-\delta^{2} / 2 \\
\cos \vartheta & =\delta \\
\sin ^{2} \vartheta & =1-\delta^{2} \\
\tan (\vartheta+\Delta) & =\frac{1-\Delta^{2} / 2-\delta^{2} / 2+\Delta \delta}{(\delta-\Delta)} \\
r & =\sqrt{a^{2}+b^{2}}-\frac{a b}{\sqrt{a^{2}+b^{2}}} \delta \\
r^{3} & =\left(a^{2}+b^{2}\right)^{3 / 2}-3 a b \sqrt{a^{2}+b^{2}} \delta+\frac{3 a^{2} b^{2}}{\sqrt{a^{2}+b^{2}}} \delta^{2}
\end{aligned}
$$

Substituting these approximations in Equation 5 and neglecting thirdorder terms in $L, \delta$, and $\Delta$ gives

$$
F^{2}=K^{2} k a b\left\{\Delta+\frac{L}{\sqrt{a^{2}+b^{2}}}\left(\frac{a b}{a^{2}+b^{2}}-\Delta+\frac{3 a^{2} b^{2} \delta}{\left(a^{2}+b^{2}\right)^{2}}\right)\right\}
$$

As in the special case described in the preceding paper, Equation 6 shows that in the general case also the period can be shortened either by increasing the angle $F D H$ in Figure 1 or by giving the spring a positive initial length. It also indicates that the period will depend least on $\delta$, or $\vartheta$, if the initial spring length is made zero, and the period is adjusted by tilting the seismograph frame.

An actual seismograph of the special type having $a=b$ has been described in the preceding paper. With it periods of over a minute were obtained, and for a period of 37 seconds the period was constant to one second for a variation of $9^{\circ}$ in the angle between the seismograph arm and the horizontal.

California Institute of Technology

Pasadena, California 